\title{
Is mesh fixation in TAPP and TEP still necessary?
}

\author{
René H. Fortelny \\ Department of General Surgery, Medical Faculty, Sigmund Freud PrivateUniversity, Vienna A1020, Austria. \\ Correspondence to: Dr. René H. Fortelny, Department of General Surgery, Head of General Surgery, Medical Faculty, Sigmund \\ Freud PrivateUniversity, Freudplatz 3, A1020 Vienna, Austria. E-mail: dr.fortelny@gmail.com
}

How to cite this article: Fortelny RH. Is mesh fixation in TAPP and TEP still necessary? Mini-invasive Surg 2021;5:16. https://dx.doi.org/10.20517/2574-1225.2021.21

Received: 18 Feb 2021 First Decision: 25 Feb 2021 Revised: 5 Mar 2021 Accepted: 9 Mar 2021 Available online: 8 Apr 2021

Academic Editor: William W. Hope Copy Editor: Yue-Yue Zhang Production Editor: Yue-Yue Zhang

\begin{abstract}
One of the most serious complications after inguinal hernia repair is still the occurrence of chronic pain. The literature describes rates of severe chronic pain of 3\%-6\%. Laparo-endoscopic inguinal hernia repair is favored to prevent postoperative pain through a minimally invasive approach and sparing of the layers of tissue covering nerves and vessels in terms of reduced risk of damage to these structures. However, the method of fixation of the mesh is still controversial discussed. The use of these penetrating devices such as staples and staplers has been shown to often be complicated by injury to nerves and vessels and occurrence of postoperative pain. The shift to completely atraumatic fixation using adhesives (fibrin glue, cyanoacrylate) began in the early part of this century. Several studies confirmed less postoperative pain after mesh fixation by glue compared to stapler or tacker. Historically, the TEP technique has always been performed without any fixation. Several studies comparing fixation versus non-fixation have been performed in TEP repair and found results with no increase in recurrence rate. Notwithstanding that very few studies comparing fixation versus no fixation with exclusion of large medial inguinal hernias have been published on this topic in TAPP repair, identical results to those with TEP repair were obtained. On the basis of current evidence, no mesh fixation is recommended for laparo-endoscopic inguinal hernia repair except for large medial and combined inguinal hernias. If mesh fixation is required, atraumatic techniques should be used.
\end{abstract}

Keywords: Laparo-endoscopic inguinal hernia repair, TAPP, TEP, mesh fixation, non-fixation, atraumatic fixation, glue fixation

\section{INTRODUCTION}

Since the introduction of minimally invasive techniques in inguinal hernia surgery with $\mathrm{TAPP}^{[1]}$ and TEP ${ }^{[2]}$, 
mesh fixation has become more and more the focus of discussion. Initially, recurrence rates were the focus of interest, but now the chronic pain rate, which is much higher in percentage terms, is assessed as a measure of surgical success. The type of fixation is closely related to the occurrence of postoperative pain. While penetrating fixation modules such as staplers and staple clips were common in the first era of TAPP and TEP, adhesive techniques have become increasingly popular. This article deals with the background of this development as well as the latest scientific published data and international guidelines and resulting tips and tricks of mesh fixation in laparo-endoscopic inguinal hernia surgery.

Since the implementation of minimal invasive techniques in inguinal hernia repair by TAPP and TEP, the discussion of mesh fixation is still controversially discussed. In the early 1990s, the standard mesh fixation in laparo-endoscopic inguinal hernia repair was performed with staples and tacks ${ }^{[3]}$. The use of these penetrating devices was frequently complicated by injury of nerves and vessels and occasionally followed by postoperative pain. Knowledge in terms of the anatomical areas of high risk for injuries to nerves and vessels such as the triangle of doom and pain are well known, but due to the variability of the nerve courses in the region of the inguinal region, a residual risk of injury in the context of a penetrating mesh fixation cannot be ruled out.

In the last two decades, therefore, the mesh fixation techniques have been under discussion. New absorbable fixation models as well as self-fixing meshes have been developed. The advantages of this atraumatic mesh fixation have been investigated in numerous studies and can be found as an evidence-based recommendation in today's guidelines for inguinal hernia care. The completely fixation-free mesh implantation in the TEP technique has been practiced by many TEP surgeons for years because of the extraperitoneal access and the fixation of the mesh resulting from the intraperitoneal pressure immediately after decompression of the pneumoperitoneum. For the TAPP technique, however, there are only a few clinical studies to date. The exact background of the advantages of atraumatic fixation techniques and also fixation-free mesh implantation will be examined in this review.

\section{Pain as main issue in inguinal hernia repair}

The recurrence rates after laparo-endoscopic hernia repair have been found to be similar to those with the open mesh techniques, especially with the standard Lichtenstein technique. However, the advantage of lower pain incidence after TAPP and TEP compared to Lichtenstein repair became apparent very soon. In a recently published meta-analysis and trial sequential analysis of primary unilateral uncomplicated inguinal hernias comparing open versus laparo-endoscopic mesh repair, the current situation of postoperative pain and recurrence was described at length and analyzed in detail ${ }^{[4]}$. This study enrolled 12 randomized controlled trials (RCT) with 3966 patients randomized to Lichtenstein repair $(n=1926)$ or laparoscopic repair $(n=2040)$. No significant differences were detected in recurrence rates between the laparoscopic and open groups [odds ratio (OR) 1.14, 95\%CI: 0.51-2.55, $P=0.76$ ]. Laparo-endoscopic repair was associated with reduced rate of acute pain compared to open repair (mean difference $1.19,95 \%$ CI: - 1.86 to - $0.51, P \leq$ 0.0006 ) as well as reduced chronic pain compared to open (OR 0.41, 95\%CI: $0.30-0.56, P \leq 0.00001$ ). A trial sequential analysis found that further studies are unlikely to demonstrate a statistically significant difference between the two techniques. This meta-analysis concluded that laparo-endoscopic repair has a statistically significant advantage in inguinal hernia repair in comparison to open mesh repair in terms of postoperative pain, and it complies with the current Hernia Surge Guidelines.

\section{Why traumatic mesh fixation in laparo-endoscopic inguinal hernia repair should be abandoned?}

In the early 1990s, the laparoscopic techniques of TAPP and TEP were developed. In addition to the discussion about mesh, questions with regard to size and fixation with a stapler or tacker were standard. The only recommendation at that time was to avoid the region caudal to the ilio-pubic tract, known as the 
triangle of doom and pain, for penetrating fixation modules ${ }^{[1]}$.

Since Reinpold's anatomical study ${ }^{[5]}$, we know that the entry points of the genital and femoral branch of the genitofemoral nerve have a great variability and can lie above the ilio-pubic tract. This widens both the triangle of doom and the triangle of pain. This also significantly increases the risk of nerve injury when using penetrating fixation models. Similarly, the varied course of the ilio-hypogastric nerve, which can also be injured during traumatic mesh fixation in TEP and TAPP repair, must also be taken into account.

In conclusion, a significantly increased risk of injury must be calculated for traumatic mesh fixation techniques, which leads to the fact that only atraumatic mesh fixation methods are recommended in the international guidelines for the laparo-endoscopic treatment of inguinal hernias ${ }^{[6]}$.

\section{Is the use of resorbable tacker able to prevent chronic postoperative pain?}

Initiated by the results of comparative studies on different mesh fixation devices, the hypothesis arose that the re-absorbability of penetrating staples could solve the problem of nerve damage, starting from neuropraxia to total dissection. By definition, the chronicity of pain appears after 3-6 months at the latest and is compared to the resorption time of these fixation models of 6-8 months. Thus, this consideration was based on lacking knowledge of the time course of a nerve injury and entrapment. Moreover, the configuration of these resorbable staples was partly incompatible with regard to the size of the mesh pores to be considered and the depth of penetration especially in laparoscopic incisional and ventral hernia repair.

In summary, the problem of penetrating fixation models is not solved by the absorbability of the material used $^{[7,8]}$ and makes no difference in outcome results such as postoperative pain and recurrence.

\section{Seroma}

The incidence of postoperative seroma formation in laparo-endoscopic inguinal hernia surgery is reported in the literature to be between 3.0 and $8 \%$ for TAPP and between $0.5 \%$ and $12.3 \%$ for TEP. A clinical association was reported with large hernia sacs in direct and indirect inguinal hernias but also with mesh fixation ${ }^{[0]}$. In a registry study by Köckerling et al. ${ }^{[10]}$, the occurrence of seroma formation after TAPP treatment was analyzed in relation to the type of fixation and the type of hernia. In the multivariate analysis, adhesive fixation had a twofold risk of postoperative seroma formation compared to staple fixation and a 5fold risk compared to non-fixation. In relation to hernia defect, M3 (direct inguinal hernia, defect size $\geq 3$ $\mathrm{cm}$, EHS classification) had a 2.8-fold increased risk compared to M1 (direct inguinal hernia, defect size $\leq$ $1.5 \mathrm{~cm}$, EHS classification) inguinal hernia, and direct inguinal hernia had a 1.2-fold increased risk compared to indirect inguinal hernia.

The closure of the direct inguinal hernia defect area of the type MIII inguinal hernia by means of inversion of the dilated transversalis fascia within laparo-endoscopic hernia repair to avoid postoperative seroma formation seems recommendable. The use of barbed suture material for this purpose seems to be suitable. The results of a RCT by Zhu et al. ${ }^{[11]}$ showed a significantly reduced incidence and volume of seroma formation without increasing the risk of recurrence, acute and chronic pain.

In another prospective study by Usmani et al. ${ }^{[12]}$ comparing direct defect closure in MII and MIII inguinal hernias by barbed non-resorbable suture versus non-closure in TEP and TAPP repair demonstrated a statistically significant reduction not only in seroma formation $(12.6 \% v s .6 .4 \%, P=0.045)$ but also in recurrence $(4.4 \% v s .0 .9 \%, P=0.036)$ after a follow-up of at least 9 months. 
The advantage of direct defect closure in prevention of recurrence was also reported in a retrospective study by Ng et al. ${ }^{[13]}$ in TAPP and TEP repair using interrupted non-resorbable single sutures for MII and MIII inguinal hernias with a $6.4 \%$ recurrence rate in the non-closure group $v s .0 \%$ in the closure group after 1 year. In both studies ${ }^{[12,13]}$, besides the defect closure, mesh fixation was performed by resorbable tacker.

In another prospective study by Clout et al. ${ }^{[14]}$ patients were treated with Endoloop closure by long term absorbable suture for MII or MIII direct defects in TEP repair. The meshes were fixated using fibrin sealant only. After a median follow-up of 5.9 years, there was no recurrence.

In summary, no mesh fixation clearly has the lowest seroma rate in laparo-endoscopic inguinal hernia surgery. But most of the studies with non-fixation of mesh excluded large direct inguinal hernias.

The defect closure respectively reducing the dilated transverse fascia by suture in MII and MII direct inguinal hernia in combination with mesh fixation by tacker or glue seems to prevent not only postoperative seroma formation but moreover the risk of recurrence.

Since atraumatic mesh fixation reduces the risk of postoperative pain, the combination of defect closure and mesh fixation by glue or the use of self-fixing meshes in MII and MIII direct inguinal hernias seems recommendable.

\section{GLUE FIXATION IN TEP AND TAPP}

\section{Fibrin glue}

Starting with the first experimental study by Katkhouda et al. ${ }^{[15]}$ by using fibrin glue for mesh fixation in TEP repair, atraumatic fixation was born. Fibrin glue, known as Tissel or Tissucol' (Baxter Healthcare, Deerfield, IL, USA), is a biologic hemostatic agent consisting of human fibrinogen and thrombin. In an experimental study, Schwab et al. ${ }^{[16]}$ carried out a biomechanical analysis of mesh fixation in TAPP and TEP comparing non-fixation versus suture versus fibrin sealant fixation. Glue fixation obtained the highest stress resistance compared to non-fixation and suture fixation. Regarding the application of fibrin sealant in laparo-endoscopic inguinal hernia repair, a spray-application at 1.5 bar pressure and a dose of approximately $0.014 \mathrm{~mL} / \mathrm{cm}^{2}$ to achieve a thin layer with broad coverage of mesh and efficient trans-porous contact with the underlaying tissue is recommended ${ }^{[17]}$.

After the first clinical publication by Langrehr et al. ${ }^{[18]}$ in 2005, several RCTs followed with fibrin fixation of mesh versus stapler and tacker fixation techniques in TAPP ${ }^{[18-22]}$ and $\mathrm{TEP}^{[23-26]}$ surgeries. The rate of postoperative pain was predominantly significantly lower compared to the penetrating fixation techniques without increased recurrence rates.

The systematic review and meta-analysis comparing fibrin glue versus staple mesh fixation in TAPP by Shi et al. ${ }^{[27]}$ including four RCTs detected no significant difference in hernia recurrence OR 2.10, 95\%CI: 0.61 7.22), seroma or hematoma formation (OR $0.55,95 \% \mathrm{CI}$ : 0.27 to 1.14 ) and operating time (SMD $0.80,95 \% \mathrm{CI}$ : -0.34 to 1.94$)$. Another systematic review and meta-analysis, by Sajid et al. ${ }^{[28]}$, with the inclusion of 5 RCTs found no significant difference regarding operating time, postoperative pain, postoperative complication, length of hospital stay and risk of recurrence, but a lower risk of chronic pain.

Kaul et al. ${ }^{[2]]}$ published a systematic review and meta-analysis comparing fibrin glue and staple fixation in TEP. In the four enrolled studies, no difference in inguinal hernia recurrence with fixation of mesh by staples/tacks versus fibrin glue (OR 2.13; 95\%CI: 0.60-7.63) was found. The incidence of chronic pain at 3 
months was significantly higher with staple/tack fixation (OR 3.25; 95\%CI: 1.62-6.49). Whereas no significant difference was seen in operative time, seroma formation, hospital stay, or time to return to normal activities.

In summary the use of fibrin glue for mesh fixation in TAPP and TEP is a safe atraumatic fixation technique and provides less chronic pain incidence compared to traumatic fixation.

The optimal application method is the spray technique to generate a thin adhesive layer.

\section{Cyanoacrylates}

Besides the biological fibrin glue, a synthetic cyanoacrylate (CA) is an alternative glue material. One of the most serious problems of the surgical use of CAs involves its degradation and toxicity. The main toxic products released by the degradation of CA alkyl chains are formaldehyde. A second basic problem associated with CAs is the flexibility. After polymerization, these polymers become hard and brittle, which might be counterproductive for tissue conditions ${ }^{[30]}$. In an in vivo preclinical study by Pascual et al.$^{[31]} \mathrm{CAs}$ currently used in clinical practice, with different alkyl chain lengths, Ifabond (n-hexyl), Glubran (n-butyl), and OCA (n-octyl) obtained sufficient tissue integration, proper mesh fixation and effective short-term biocompatibility. CA (n-octyl) revealed the lowest seroma formation macrophage response.

The largest number of mesh fixations by CAs (n-butyl) in TAPP repair was published by Kukleta et al. ${ }^{[32]}$ showing excellent results in terms of biocompatibility and risk of recurrence. The technique recommended by these authors for CA mesh fixation consists in applying just a few drops each to all four quadrants of the mesh. Subwongcharoen et al. ${ }^{[33]}$ reported on a RCT comparing staple fixation versus CA (n-butyl) in TEP repair. Postoperative pain assessed by VAS was significantly higher in the staple group after 24 hours (1.6 $+/$ - 1.33 vs. $2.35+/$ - 1.32) $(P=0.037)$. The rate of chronic pain after 3 months and 1 year was higher in the staple group but did not reach significance. Complications rates and recurrences after one year were not significant.

In summary, cyanoacrylate, preferably n-octyl cyanoacrylate, is safe to use for adhesive fixation of meshes in TAPP and TEP. Care should be taken to ensure sparing spot application. This is in contrast to the large-area trans-porous spray application of fibrin glue, which achieves elastic fixation of the fibrin glue ${ }^{[34]}$.

\section{Self-fixating mesh}

So far, in contrast to the open mesh methods, there are only a very few publications for the use of selffixating mesh. In feasibility studies with the use of self-fixation mesh in TAPP by Birk et al. ${ }^{[35]}$ and Li et al. ${ }^{[36]}$ in TAPP and TEP and by Bresnahan et al. ${ }^{[37]}$ in TEP, only one RCT by Denham et al. ${ }^{[38]}$ was published in 2019. In this study 217 patients with primary, unilateral inguinal hernias were randomized to non-selffixation or self-fixation group in TEP repair. A subgroup randomization was performed on the self-fixating mesh group with direct hernias $>2 \mathrm{~cm}(n=38)$. Fifty percent of this group $(n=19)$ were randomized to receive tacker fixation. The median operative times and length of hospital stay were similar. More patients in the non-fixating mesh group received tacks (43 vs. 19, $P=0.001$ ). During the first 3 postoperative days non-fixating mesh patients reported significantly less pain, whereas 3 weeks or 1 year postoperatively no significant difference was detected. In the follow-up of one year, no recurrence was found in either of the groups. A subgroup analysis of direct inguinal hernias could not be performed due to the low number of patients. In conclusion, the authors stated that "self-fixating mesh does not appear to positively impact QoL after TEP repair". 
Since the evidence regarding the benefit using self-fixating meshes in laparo-endoscopic inguinal hernia repair is too little, no conclusions or recommendations can be derived at present.

\section{Is there a need for mesh fixation in TEP or TAPP?}

Finally, the main topic of this paper is the discussion of non-fixation of mesh in laparo-endoscopic inguinal hernia surgery.

The first study in terms of non-fixation in TEP repair in an experimental setting by Katkhouda et al. ${ }^{[15]}$ demonstrated the risk of mesh movement. Despite this finding and an increased potential risk of early recurrence derived from it, the non-fixation in TEP technique, for the difference of TAPP, has been thematized very early. The obvious reason for this was the specific technique of implanting the mesh in a pocket that made it unlikely that the mesh would slip after the pneumoperitoneum was depressurized. On the other hand, the advantage of not fixing the meshes is associated with a significantly reduced risk of seroma occurrence ${ }^{[10]}$.

In a study by Claus ${ }^{[39]}$ specifically focused on mesh displacement in the absence of fixation in TEP repair, only a minimal displacement was found. The comparison of radiologically controlled mesh movement after bilateral versus unilateral TEP repair showed 30 days postoperatively a median of 1.9 and $1.8 \mathrm{~cm}(P=0.78)$, respectively. With this aspect of potential, albeit minor mesh displacement, care must be taken to ensure adequate size and defect overlap, especially in large direct inguinal hernias.

In 1999, Ferzli et al ${ }^{[40]}$ published the first study comparing tacker versus non-fixation in TEP repair without significant differences in recurrence or complication rates after a 12-month follow-up.

Since then, several studies ${ }^{[41-46]}$ and meta-analyses ${ }^{[47-49]}$ of TEP procedures with non-fixation were published. The conclusion of these were that outcomes after non-fixation in TEP repair are comparable to fixation and not associated with higher recurrence rates. However, the various meta-analyses had a certain bias due to the inclusion of RCTs with recurrent surgery, bilateral inguinal hernias, both sexes and exclusion of large medial inguinal hernias. As there has been no RCT on primary unilateral inguinal hernias to date, an evidence-based statement can only be drawn to a limited extent.

Based on the Swedish Hernia Registry, a study including 1110 male patients undergoing TEP repair comparing permanent fixation versus non-fixation including glue fixation in terms of chronic pain detected no significant difference ${ }^{[50]}$. Going into detailed analyses, the rate of permanent fixation was significantly higher in medial hernias compared to non-fixation and glue fixation $(P<0.003)$ as well as regarding the defect size $(P<0.002)$. The distribution of unilateral inguinal to bilateral and recurrent inguinal hernias was 36,64 and $9 \%$, respectively. The use of heavy meshes were significantly more frequent in the fixation group compared to non-fixation and glue-fixation $(P<0.015)$. In a subgroup-analysis, the use of glue fixation was performed significantly more in medial hernia compared to non-fixation $(P<0.001)$. After a median followup of 7.5 years, a total of 15 patients had an operation for recurrent hernia: $1.5 \%$ for fixation and $1.3 \%$ for non-fixation and glue-fixation $(P<0.735)$. Looking to the sub-analysis of recurrences after medial hernia repair, no significant difference was seen $(0.7 \%$ after fixation $v$ s. $1.7 \%$ after non-fixation and glue-fixation; $P$ $<0.669)$. In a multivariable analysis, the risk factor for chronic pain was a postoperative complication.

In summary, in this registry study of TEP repair in male patients, a low incidence of recurrence was observed with no significant difference seen in non fixation, permanent and glue fixation. The conclusion of this study suggests that non-fixation in TEP repair does not carry a risk of recurrence even in medial 
hernias. Nevertheless, the subgroup analysis shows that glue-fixation in medial hernias was significantly more frequently used compared to non-fixation. Since no evaluation is available regarding the size and type of fixation of medial inguinal hernias, the interpretation in this regard should also be viewed with caution.

For the TAPP procedure, only $2 \mathrm{RCTs}^{[51,52]}$ comparing non-fixation with staple fixation have been published to date. In 1999, Smith et al. ${ }^{[51]}$ reported a recurrence rate after median follow-up of 16 months (range, 1-32 months) of $0 \%$ after non-fixation and $1 \%$ after staple fixation without significant difference $(P=0.09)$. Furthermore, no significant difference was detected in operative time and chronic pain between the two groups. Limitations of this study have to be considered regarding the short time of follow up and the number of patients followed up of only $65 \%$ by examination and $22.2 \%$ by telephone. In the study by Li et al. ${ }^{[52]}$, male patients with primary, unilateral inguinal hernia, defect size $<4 \mathrm{~cm}$ diameter were randomly allocated to non-fixation or staple fixation. After a median follow up of 11.5 months after non-fixation and 11.2 months after staple fixation, no recurrences were found. Postoperative VAS pain scores up to 6 months for the non-fixation group were significantly lower than in the fixation group. The quality of life regarding physical function, physical role, bodily pain, and general health in the non-fixation group was significantly better than in the fixation group. This RCT also had limitations regarding the very short follow-up period and the inclusion criterion restricted to smaller than 4 -cm defect size.

In summary, the question of non-fixation in TEP and TAPP has limited answerability. The lowest common denominator for low-risk non-fixation of meshes in TEP and TAPP techniques seems to be primary unilateral male inguinal hernias with exclusion of medial hernia types with a defect diameter of $\geq 3 \mathrm{~cm}$.

\section{Discussion}

The appropriate technique in TEP and TAPP repair is the most important requirement of prevention of postoperative pain. The dissection in the right plane with preserving the protective layers such as spermatic sheath to prevent nerve injury and to avoid any coarse grasp of the spermatic cord are basic rules to be observed. The preparation of the landing zone has to be sufficient for a mesh implantation of at least $10 \mathrm{~cm}$ by $15 \mathrm{~cm}$. In the special case of direct hernia with a defect size of $3 \mathrm{~cm}$ and more the mesh size has to be larger, e.g., $12 \mathrm{~cm}$ by $17 \mathrm{~cm}$ to guarantee a sufficient overlap of at least $3 \mathrm{~cm}$ over the midline. In addition, the inversion of the dilated transverse fascia seems to prevent postoperative seroma occurrence in these cases. Following these crucial steps of TEP or TAPP are mandatory to achieve best outcomes regarding postoperative pain and recurrence rate.

The choice of the optimal mesh for laparo-endoscopic inguinal hernia repair has been discussed for years with the question of light or heavy weight. Until recently, lightweight meshes were clearly preferred in terms of pain and reduced foreign body reaction, but an RCT with long-term results has changed the evidence ${ }^{[53]}$. In this 5-year follow-up RCT study in TEP repair of primary unilateral inguinal hernias, the recurrence rate was significantly increased after the use of lightweight mesh (UltraPro ) compared to the use of heavyweight mesh. This publication did not remain uncommented ${ }^{[54]}$. Since the classification of medial hernias in this study is based on the Nyhus classification ${ }^{[5]}$ and not on the EHS classification ${ }^{[56]}$ with differentiation of defect sizes (MI, MII and MII), the MIII hernia cannot be evaluated selectively.

The studies, already mentioned in the seroma chapter regarding the closure or shortening of the dilated transverse fascia have not only led to a reduction in seroma formation but also to a decrease of the recurrence rate. This relationship seems quite plausible. Considering the bending stiffness of small poresized/heavyweight meshes compared to large pore-sized/lightweight meshes, significant differences can be found, which are especially important when there is no tissue directly under the mesh but an empty space. 
In the biomechanical study by Hollinsky et al. ${ }^{[57]}$, the ultimate tensile strength and elasticity in association with defect size of $1.5,3$ and $5 \mathrm{~cm}$ was assessed by the use of a lightweight mesh in comparison to a heavyweight mesh. Regarding $1.5 \mathrm{~cm}$ of defect size no difference was seen, but in case of defect size 3 and 5 $\mathrm{cm}$, the lightweight mesh flexed $3.16+/-0.4 \mathrm{~mm}$ and $10.4+/-2.5 \mathrm{~mm}$ significantly more in comparison to the heavyweight mesh $0.34+/-0.2 \mathrm{~mm}$ and $3.97+/-0.7 \mathrm{~mm}(P<0.001)$. This study is of main importance to understand biomechanical relationships of mesh properties and defect size.

While meta-analyses to date have shown advantages for the use of lightweight meshes in laparo-endoscopic inguinal hernia surgery ${ }^{[58]}$, the inclusion of the TEP study by Ross et al.${ }^{[53]}$ changed the recommendation not to use lightweight meshes, especially in direct hernias, due to the increased risk of recurrence (RR 2.21; 95\%CI: 1.14-4.31), especially in non-fixated mesh direct repairs (RR 7.27; 95\%CI: 1.33-39.73) and/or large hernia defects ${ }^{[59]}$. No significant differences were determined in terms of pain and foreign body sensation. Similar results were found in the meta-analysis of $\mathrm{Hu}$ et al. ${ }^{[60]}$.

If you look at the EHS update guidelines from $2014^{[61]}$, you will find the recommendations for mesh fixation in TEP if a heavyweight mesh is used: traumatic mesh fixation should be avoided except in large direct inguinal hernias. For TAPP treatment, atraumatic mesh fixation without increased risk of recurrence within one year was recommended.

Nowadays, in the nomenclature of mesh properties, light and heavy are obsolete; rather, effective porosity and surface properties as well as elasticity are some of the defining properties of meshes. However, there is a complex interplay between the polymer, textile structure, amount of material, porosity, processing of the material, position and mechanical load on the mesh.

Despite some limitations of the available evidence, the HerniaSurge Group stated in the current guidelines ${ }^{[6]}$ that mesh fixation is not required in almost all types of inguinal hernias in TEP repair. However, a strong recommendation for mesh fixation was made for large medial inguinal hernias (MIII in the EHS classification) for TAPP and TEP repair. If fixation is required, the HerniaSurge guidelines recommend an atraumatic technique to reduce the risk of early postoperative pain.

In a Herniamed register study, 11,228 male patients with primary unilateral inguinal hernia underwent TAPP technique and were followed up for 1 year. In this study published by Mayer et al ${ }^{[62]}$, mesh fixation was performed in a total of $66.1 \%$ of the procedures. In the unadjusted analysis, there was no significant difference in recurrence rate $(0.88 \%$ with fixation $v s .1 .1 \%$ without fixation; $P=0.259)$. In a multivariable analysis of all potential influencing factors such as age, ASA, BMI, risk factors, defect size, mesh fixation, location of the defect and mesh size, no factor was identified to influence the recurrence rate at 1-year follow-up. However, for medial and combined defect localization in comparison to lateral localization, a highly significant effect was detected $(P<0.001)$. Using mesh fixation and larger meshes, it was possible to significantly reduce the recurrence rate for larger medial hernias in this series $(P=0.046)$. This registry study clearly confirms the need of mesh fixation for MIII inguinal hernias, as recommended by the HerniaSurge Guidelines, but also for combined inguinal hernias and impressively demonstrates the advantage of using larger implants for recurrence prevention.

\section{CONCLUSIONS}

The central question of fixation or non-fixation of mesh in laparo-endoscopic inguinal hernia management can only be viewed and answered on a multifactorial basis. According to the existing literature, it is recommended that mesh fixation should be performed in case of medial as well as combined inguinal 
hernias. The inversion of dilated parts of the transverse fascia in M III inguinal hernias to prevent the formation of seroma and recurrence, as well as the implantation of larger meshes, also seems to be preferable in this constellation. In contrast, the use of ultra-lightweight, large-pored meshes without mesh fixation does not seem to be appropriate in this indication. For all other types of inguinal hernias, mesh fixation can be omitted but always under the condition that all standards of laparo-endoscopic inguinal hernia management are met.

\section{DECLARATIONS}

\section{Authors' contributions}

All substantial contributions to the concept and design of this review, performed literature search, interpretation and conclusions: Fortelny RH

\section{Availability of data and materials}

Not applicable.

\section{Financial support and sponsorship}

None.

\section{Conflicts of interest}

Dr. Fortelny reports receipt of honoraria from BD BARD and B.Braun for speaking services.

\section{Ethical approval and consent to participate}

Not applicable.

\section{Consent for publication}

Not applicable.

\section{Copyright}

(c) The Author(s) 2021.

\section{REFERENCES}

1. Arregui ME, Navarrete J, Davis CJ, Castro D, Nagan RF. Laparoscopic inguinal herniorrhaphy. Techniques and Controversies. Surg Clin North Am 1993;73:513-27. DOI PubMed

2. Ferzli G, Sayad P, Huie F, Hallak A, Usal H. Endoscopic extraperitoneal herniorrhaphy. A 5-year experience. Surg Endosc 1998;12:1311-3. DOI PubMed

3. Bittner R, Leibl B, Kraft K, Däubler P, Schwarz J. Die laparoskopische Hernioplastik (TAPP)-Komplikationen und Rezidive bei 900 Operationen [Laparoscopic hernioplasty (TAPP)-complications and recurrences in 900 operations]. Zentralbl Chir 1996;121:313-9. PubMed

4. Bullen NL, Massey LH, Antoniou SA, Smart NJ, Fortelny RH. Open versus laparoscopic mesh repair of primary unilateral uncomplicated inguinal hernia: a systematic review with meta-analysis and trial sequential analysis. Hernia 2019;23:461-72. DOI PubMed

5. Reinpold W, Schroeder AD, Schroeder M, Berger C, Rohr M, Wehrenberg U. Retroperitoneal anatomy of the iliohypogastric, ilioinguinal, genitofemoral, and lateral femoral cutaneous nerve: consequences for prevention and treatment of chronic inguinodynia. Hernia 2015;19:539-48. DOI PubMed

6. $\quad$ guidelines for groin hernia management. Hernia 2018;22:1-165. DOI PubMed PMC

7. Prakash PS, Wijerathne S, Salgaonkar HP, Lomanto D. The efficacy of absorbable versus non-absorbable fixation in laparoscopic totally extraperitoneal (tep) repair of large inguinal hernias. Asian J Surg 2019;42:995-1000. DOI PubMed

8. Christoffersen MW, Brandt E, Helgstrand F, et al. Recurrence rate after absorbable tack fixation of mesh in laparoscopic incisional hernia repair. Br J Surg 2015;102:541-7. DOI PubMed

9. Garg P, Rajagopal M, Varghese V, Ismail M. Laparoscopic total extraperitoneal inguinal hernia repair with nonfixation of the mesh for 1,692 hernias. Surg Endosc 2009;23:1241-5. DOI PubMed

10. Köckerling F, Bittner R, Adolf D, et al. Seroma following transabdominal preperitoneal patch plasty (TAPP): incidence, risk factors, and preventive measures. Surg Endosc 2018;32:2222-31. DOI PubMed PMC

11. Zhu Y, Liu M, Li J, Wang M. Closure of Direct Inguinal Hernia Defect in Laparoscopic Hernioplasty to Prevent Seroma Formation: A 
Prospective Double-blind Randomized Controlled Trial. Surg Laparosc Endosc Percutan Tech 2019;29:18-21. DOI PubMed

12. Usmani F, Wijerathne S, Malik S, Yeo C, Rao J, Lomanto D. Effect of direct defect closure during laparoscopic inguinal hernia repair ("TEP/TAPP plus" technique) on post-operative outcomes. Hernia 2020;24:167-71. DOI PubMed

13. Ng AY, Lin J, Ching SS, Lee J, Wong ASY. Does primary closure of direct inguinal hernia defect during laparoscopic mesh repair reduce the risk of early recurrence? Hernia 2020;24:1093-8. DOI PubMed

14. Clout E, Thayaparan M, Douglas C, Berney CR. Long-term follow-up of endoscopic totally extraperitoneal direct inguinal hernia repair using the Endoloop technique. Surg Endosc 2019;33:2967-74. DOI PubMed

15. Katkhouda N, Mavor E, Friedlander MH, et al. Use of fibrin sealant for prosthetic mesh fixation in laparoscopic extraperitoneal inguinal hernia repair. Ann Surg 2001;233:18-25. DOI PubMed PMC

16. Schwab R, Schumacher O, Junge K, et al. Biomechanical analyses of mesh fixation in TAPP and TEP hernia repair. Surg Endosc 2008;22:731-8. DOI PubMed

17. Brand J, Gruber-Blum S, Gruber K, Fortelny RH, Redl H, Petter-Puchner AH. Transporous hernia mesh fixation with fibrin sealant in an in vitro model of spray application. J Surg Res 2013;183:726-32. DOI PubMed

18. Langrehr JM, Schmidt SC, Neuhaus P. Initial experience with the use of fibrin sealant for the fixation of the prosthetic mesh in laparoscopic transabdominal preperitoneal hernia repair. Rozhl Chir 2005;84:399-402. PubMed

19. Lovisetto F, Zonta S, Rota E, et al. Use of human fibrin glue (Tissucol) versus staples for mesh fixation in laparoscopic transabdominal preperitoneal hernioplasty: a prospective, randomized study. Ann Surg 2007;245:222-31. DOI PubMed PMC

20. Olmi S, Erba L, Bertolini A, Scaini A, Croce E. Fibrin glue for mesh fixation in laparoscopic transabdominal preperitoneal (TAPP) hernia repair: indications, technique, and outcomes. Surg Endosc 2006;20:1846-50. DOI PubMed

21. Fortelny RH, Petter-Puchner AH, May C, et al. The impact of atraumatic fibrin sealant vs. staple mesh fixation in TAPP hernia repair on chronic pain and quality of life: results of a randomized controlled study. Surg Endosc 2012;26:249-54. DOI PubMed

22. Tolver MA, Rosenberg J, Juul P, Bisgaard T. Randomized clinical trial of fibrin glue versus tacked fixation in laparoscopic groin hernia repair. Surg Endosc 2013;27:2727-33. DOI PubMed

23. Katkhouda N. A new technique for laparoscopic hernia repair using fibrin sealant. Surg Technol Int 2004;12:120-6. PubMed

24. Lau H. Fibrin sealant versus mechanical stapling for mesh fixation during endoscopic extraperitoneal inguinal hernioplasty: a randomized prospective trial. Ann Surg 2005;242:670-5. DOI PubMed PMC

25. Novik B, Hagedorn S, Mörk UB, Dahlin K, Skullman S, Dalenbäck J. Fibrin glue for securing the mesh in laparoscopic totally extraperitoneal inguinal hernia repair: a study with a 40-month prospective follow-up period. Surg Endosc 2006;20:462-7. DOI PubMed

26. Schwab R, Willms A, Kröger A, Becker HP. Less chronic pain following mesh fixation using a fibrin sealant in TEP inguinal hernia repair. Hernia 2006;10:272-7. DOI PubMed

27. Shi Z, Fan X, Zhai S, Zhong X, Huang D. Fibrin glue versus staple for mesh fixation in laparoscopic transabdominal preperitoneal repair of inguinal hernia: a meta-analysis and systematic review. Surg Endosc 2017;31:527-37. DOI PubMed

28. Sajid MS, Ladwa N, Kalra L, McFall M, Baig MK, Sains P. A meta-analysis examining the use of tacker mesh fixation versus glue mesh fixation in laparoscopic inguinal hernia repair. Am J Surg 2013;206:103-11. DOI PubMed

29. Kaul A, Hutfless S, Le H, et al. Staple versus fibrin glue fixation in laparoscopic total extraperitoneal repair of inguinal hernia: a systematic review and meta-analysis. Surg Endosc 2012;26:1269-78. DOI PubMed

30. Fortelny RH, Petter-Puchner AH, Walder N, et al. Cyanoacrylate tissue sealant impairs tissue integration of macroporous mesh in experimental hernia repair. Surg Endosc 2007;21:1781-5. DOI PubMed

31. Pascual G, Sotomayor S, Rodriguez M, et al. Cytotoxicity of cyanoacrylate-based tissue adhesives and short term preclinical in vivo biocompatibility in abdominal hernia repair. PLoS One 2016;11:e157920. DOI PubMed PMC

32. Kukleta JF, Freytag C, Weber M. Efficiency and safety of mesh fixation in laparoscopic inguinal hernia repair using n-butyl cyanoacrylate: long-term biocompatibility in over 1,300 mesh fixations. Hernia 2012;16:153-62. DOI PubMed PMC

33. Subwongcharoen S, Ruksakul K. A randomized controlled trial of staple fixation versus N-butyl-2-cyanoacrylate fixation in laparoscopic inguinal hernia repair. J Med Assoc Thai 2013;96 Suppl 3:S8-13. PubMed

34. Fortelny RH, Petter-Puchner AH, Glaser KS, Redl H. Use of fibrin sealant (Tisseel/Tissucol) in hernia repair: a systematic review. Surg Endosc 2012;26:1803-12. DOI PubMed

35. Birk D, Pardo CG. Self-gripping Parietene and Parietex Progrip mesh laparoscopic hernia repair: have we found the ideal implant? Surg Technol Int 2012;22:93-100. PubMed

36. Li J, Shao X, Cheng T. How I do it: the horizontal-bilateral unfolding method for self-gripping (Progrip ${ }^{\mathrm{TM}}$ ) mesh placement in laparoscopic inguinal hernia repair. Hernia 2019;23:809-15. DOI PubMed

37. Bresnahan E, Bates A, Wu A, Reiner M, Jacob B. The use of self-gripping (Progrip ${ }^{\mathrm{TM}}$ ) mesh during laparoscopic total extraperitoneal (TEP) inguinal hernia repair: a prospective feasibility and long-term outcomes study. Surg Endosc 2015;29:2690-6. DOI PubMed

38. Denham M, Johnson B, Leong M, et al. An analysis of results in a single-blinded, prospective randomized controlled trial comparing non-fixating versus self-fixating mesh for laparoscopic inguinal hernia repair. Surg Endosc 2019;33:2670-9. DOI PubMed

39. Claus CMP, Rocha GM, Campos ACL, Paulin JAN, Coelho JCU. Mesh Displacement After Bilateral Inguinal Hernia Repair With No Fixation. JSLS 2017;21:e2017. DOI PubMed PMC

40. Ferzli GS, Frezza EE, Pecoraro AM Jr, Ahern KD. Prospective randomized study of stapled versus unstapled mesh in a laparoscopic preperitoneal inguinal hernia repair. J Am Coll Surg 1999;188:461-5. DOI PubMed

41. Garg P, Nair S, Shereef M, et al. Mesh fixation compared to nonfixation in total extraperitoneal inguinal hernia repair: a randomized controlled trial in a rural center in India. Surg Endosc 2011;25:3300-6. DOI PubMed 
42. Koch CA, Greenlee SM, Larson DR, Harrington JR, Farley DR. Randomized prospective study of totally extraperitoneal inguinal hernia repair: fixation versus no fixation of mesh. JSLS 2006;10:457-60. PubMed PMC

43. Lau H, Patil NG. Selective non-stapling of mesh during unilateral endoscopic total extraperitoneal inguinal hernioplasty: a case-control study. Arch Surg 2003;138:1352-5. DOI PubMed

44. Moreno-Egea A, Torralba Martínez JA, Morales Cuenca G, Aguayo Albasini JL. Randomized clinical trial of fixation vs nonfixation of mesh in total extraperitoneal inguinal hernioplasty. Arch Surg 2004;139:1376-9. DOI PubMed

45. Parshad R, Kumar R, Hazrah P, Bal S. A randomized comparison of the early outcome of stapled and unstapled techniques of laparoscopic total extraperitoneal inguinal hernia repair. JSLS 2005;9:403-7. PubMed PMC

46. Taylor C, Layani L, Liew V, Ghusn M, Crampton N, White S. Laparoscopic inguinal hernia repair without mesh fixation, early results of a large randomised clinical trial. Surg Endosc 2008;22:757-62. DOI PubMed

47. Tam KW, Liang HH, Chai CY. Outcomes of staple fixation of mesh versus nonfixation in laparoscopic total extraperitoneal inguinal repair: a meta-analysis of randomized controlled trials. World J Surg 2010;34:3065-74. DOI PubMed

48. Teng YJ, Pan SM, Liu YL, et al. A meta-analysis of randomized controlled trials of fixation versus nonfixation of mesh in laparoscopic total extraperitoneal inguinal hernia repair. Surg Endosc 2011;25:2849-58. DOI PubMed

49. Sajid MS, Ladwa N, Kalra L, Hutson K, Sains P, Baig MK. A meta-analysis examining the use of tacker fixation versus no-fixation of mesh in laparoscopic inguinal hernia repair. Int J Surg 2012;10:224-31. DOI PubMed

50. Gutlic N, Rogmark P, Nordin P, Petersson U, Montgomery A. Impact of Mesh Fixation on Chronic Pain in Total Extraperitoneal Inguinal Hernia Repair (TEP): A Nationwide Register-based Study. Ann Surg 2016;263:1199-206. DOI PubMed

51. Smith AI, Royston CM, Sedman PC. Stapled and nonstapled laparoscopic transabdominal preperitoneal (TAPP) inguinal hernia repair. A prospective randomized trial. Surg Endosc 1999;13:804-6. DOI PubMed

52. Li W, Sun D, Sun Y, et al. The effect of transabdominal preperitoneal (TAPP) inguinal hernioplasty on chronic pain and quality of life of patients: mesh fixation versus non-fixation. Surg Endosc 2017;31:4238-43. DOI PubMed

53. Roos MM, Bakker WJ, Schouten N, et al. Higher Recurrence Rate After Endoscopic Totally Extraperitoneal (TEP) Inguinal Hernia Repair With Ultrapro Lightweight Mesh: 5-Year Results of a Randomized Controlled Trial (TULP-trial). Ann Surg 2018;268:241-6. DOI PubMed

54. Li J, Zhang W. Comments on "Higher Recurrence Rate After Endoscopic Totally Extraperitoneal (TEP) Inguinal Hernia Repair With Ultrapro Lightweight Mesh: 5-Year Results of a Randomized Controlled Trial (TULP-trial)". Ann Surg 2019;269:e38-9. DOI PubMed

55. Nyhus LM. Classification of groin hernia: milestones. Hernia 2004;8:87-8. DOI PubMed

56. Miserez M, Alexandre JH, Campanelli G, et al. The European hernia society groin hernia classification: simple and easy to remember. Hernia 2007;11:113-6. DOI PubMed

57. Hollinsky C, Sandberg S, Koch T, Seidler S. Biomechanical properties of lightweight versus heavyweight meshes for laparoscopic inguinal hernia repair and their impact on recurrence rates. Surg Endosc 2008;22:2679-85. DOI PubMed

58. Currie A, Andrew H, Tonsi A, Hurley PR, Taribagil S. Lightweight versus heavyweight mesh in laparoscopic inguinal hernia repair: a meta-analysis. Surg Endosc 2012;26:2126-33. DOI PubMed

59. Bakker WJ, Aufenacker TJ, Boschman JS, Burgmans JPJ. Heavyweight Mesh Is Superior to Lightweight Mesh in Laparo-Endoscopic Inguinal Hernia Repair: A Meta-analysis and Trial Sequential Analysis of Randomized Controlled Trials. Ann Surg 2020. DOI PubMed

60. Hu D, Huang B, Gao L. Lightweight Versus Heavyweight Mesh in Laparoscopic Inguinal Hernia Repair: An Updated Systematic Review and Meta-Analysis of Randomized Trials. J Laparoendosc Adv Surg Tech A 2019;29:1152-62. DOI PubMed

61. Miserez M, Peeters E, Aufenacker T, et al. Update with level 1 studies of the European Hernia Society guidelines on the treatment of inguinal hernia in adult patients. Hernia 2014;18:151-63. DOI PubMed

62. Mayer F, Niebuhr H, Lechner M, et al. When is mesh fixation in TAPP-repair of primary inguinal hernia repair necessary? Surg Endosc 2016;30:4363-71. DOI PubMed PMC 